\title{
Improved AcroQoL scores in acromegaly after sagittal split ramus osteotomy with Le Fort I osteotomy
}

\author{
Tomomi Taguchi ${ }^{1} 1$, Sachiko Kimizuka² and Koji Takano1 \\ 'Department of Endocrinology, Diabetes and Metabolism, Kitasato University School of Medicine and 2Department \\ of Plastic and Aesthetic Surgery, Kitasato University School of Medicine, Sagamihara, Kanagawa, Japan
}

Correspondence should be addressed to T Taguchi

Email

t.tomo@kitasato-u.ac.jp

\section{Summary}

Acromegaly is associated with a low quality of life (QoL), which is partially attributable to appearance. However, appearance changes are only partially reversible with treatments of growth hormone excess. This case study describes a 41-year-old Japanese man who presented with mandibular prognathism. Acromegaly was suspected because of the patient's facial features. Subsequent examination revealed a pituitary tumour with elevated levels of growth hormone and insulin-like growth factor 1 (IGF1), confirming a diagnosis of acromegaly. We assessed his QoL with the acromegaly QoL questionnaire (AcroQoL) before transsphenoidal surgery, and all AcroQoL scores were low. Although the pituitary adenoma was resected, his serum IGF1 levels started to increase again and MRI identified a residual pituitary lesion. After lanreotide and pegvisomant injection therapies improved his serum IGF1 levels, we reassessed his AcroQoL scores, however, the results showed worsening scores regarding appearance and personal relationships, and the patient expressed interest in surgery for mandibular prognathism. We performed sagittal split ramus osteotomy (SSRO) with Le Fort I osteotomy, thus, the patient's AcroQoL scores improved. This case shows that SSRO with Le Fort I osteotomy for mandibular prognathism, rather than control of serum IGF1 level alone, improved the patient's AcroQoL score, especially for both psychological well-being and approval of appearance. Acromegaly has many complications, including its negative impact on patients' perception of their appearance and patients' QoL can be improved using multiple options, in addition to controlling growth hormone levels.

\section{Learning points:}

- The patient's AcroQoL scores worsened despite biochemical normalization of IGF-1 levels.

- Mandibular prognathism due to acromegaly can be successfully operated by performing sagittal split ramus osteotomy with Le Fort I osteotomy.

- Frequent monitoring of AcroQoL scores and appropriate response to negative results can improve the overall QoL.

\section{Background}

Acromegaly is a chronic disease that results from an excessive production of growth hormone (GH) due to pituitary adenoma and is associated with comorbidities, such as hypertension, diabetes mellitus, heart failure, and increased mortality. Excessive GH and insulin-like growth factor 1 (IGF1) secretions also lead to acral overgrowth, jaw prognathism, and change in facial appearance (1). These comorbidities significantly affect the quality of life (QoL) of patients with acromegaly, despite achieving a biochemical control through treatment (2). The orofacial 
features in patients with acromegaly include particularly thick lips with a tendency towards mandibular overgrowth with prognathism, jaw thickening, maxillary widening, drifting of teeth, malocclusion, and increased gonial angle. Many patients with acromegaly are dissatisfied with their appearance. The acromegaly QoL questionnaire (AcroQoL) is a disease-specific QoL questionnaire that was first introduced in Spanish (3). The AcroQoL is useful to assess QoL and therapeutic effects of acromegaly treatments.

Here, we report that transsphenoidal surgery (TSS) and injection therapies for acromegaly with mandibular prognathism did not improve AcroQoL scores, but that sagittal split ramus osteotomy (SSRO) with Le Fort I osteotomy surgery could help patients achieve higher QoL scores.

\section{Case presentation}

A 41-year-old man was referred to the Endocrinology Department. He experienced enlarged hands and feet for 10 years and was alerted to facial changes by a friend. Acromegalic features such as mandibular prognathism, thick lips, and thickening of the hands and feet were observed. The patient had a history of impaired glucose tolerance for 5 years. There was no family history of acromegaly.

\section{Investigation}

He was diagnosed with diabetes mellitus (fasting plasma glucose, $190 \mathrm{mg} / \mathrm{dL}$; HbA1c, 9.0\%). His GH and IGF1 serum levels were elevated $(34.90 \mathrm{ng} / \mathrm{mL}$ and $923 \mathrm{ng} / \mathrm{mL}$ (+9.8 s.D.), respectively). A 20 -mm suprasellar tumour was detected on MRI. Bromocriptine $(2.5 \mathrm{mg}$ ) administered orally raised GH levels from $38.1 \mathrm{~g} / \mathrm{mL}$ to $59.2 \mathrm{~g} / \mathrm{mL}$, and octreotide $(50 \mu \mathrm{g})$ administered subcutaneously did not sufficiently suppress $\mathrm{GH}$ levels from $55.0 \mathrm{ng} / \mathrm{mL}$ to $16.8 \mathrm{ng} / \mathrm{mL}$. We did not perform the $75 \mathrm{~g}$ oral glucose tolerance test because of his severe diabetes mellitus. The patient was diagnosed with acromegaly based on the diagnostic guidelines (4). Moreover, we assessed his AcroQoL scores (total score, 59\%; physical score, 71.9\%; and psychological score, 51.8\%). Subscale appearance and personal relationship scores were $35.7 \%$ and $67.9 \%$, respectively (Fig. 1).

\section{Treatment}

TSS was performed to treat the excessive GH production. After the procedure, serum GH levels did not decline below

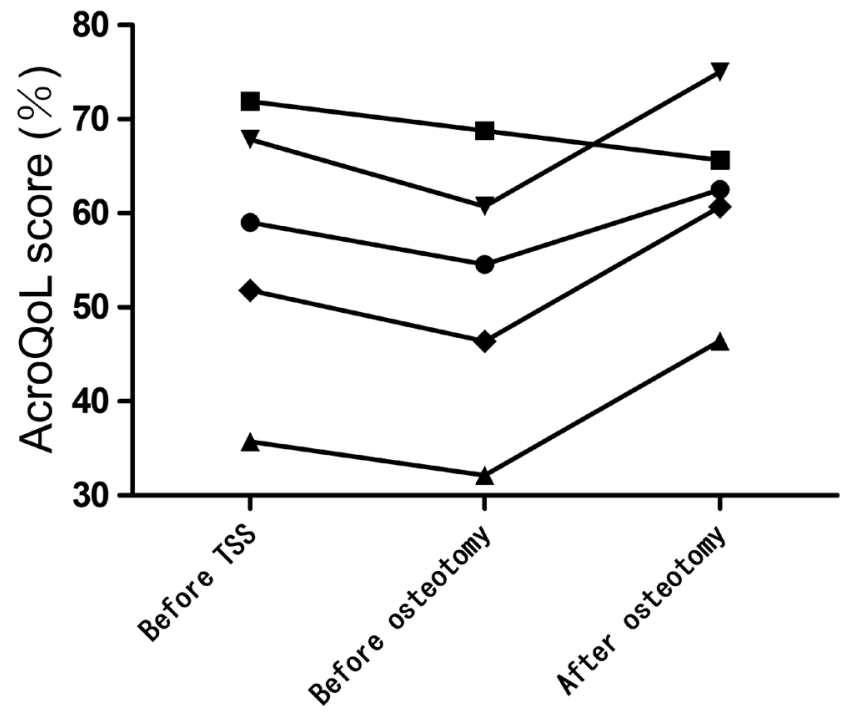

Figure 1

Graph showing changes in the patient's AcroQoL scores. Closed circle, total scores; closed square, physical scores; closed diamond, psychological scores; closed triangle, appearance scores; closed reverse triangle, personal relationship score.

the normal range (75 g oral glucose tolerance test: nadir $\mathrm{GH}, 1.98 \mathrm{ng} / \mathrm{mL}$; normal range $<0.4 \mathrm{ng} / \mathrm{mL}$ ). Pathological analysis showed a dark eosinophilic adenoma with diffuse positivity for GH and prolactin. CAM5.2 staining showed sparsely granulated adenoma, and the Ki67 labelling index was approximately $0.3 \%$. Four months after the TSS, the serum IGF1 level was elevated to $300 \mathrm{ng} / \mathrm{mL}$ (normal range 94-241 ng/mL, S.D. +2.7) and the patient experienced sweating. MRI showed the residual lesion. Treatment was initiated with lanreotide $120 \mathrm{mg}$ administered monthly and pegvisomant $15 \mathrm{mg}$ administered every other day, until the normalization of his serum IGF1 level; the treatment was continued after normalization.

The patient complained of his facial appearance, however, which was completely different from his appearance as a youth. He hoped to undergo cosmetic surgery for mandibular prognathism to recover his previous appearance (Fig. 2A and B). His facial conditions were mandibular prognathism and opposite occlusion. After his serum IGF1 levels were normalized with the medication therapies, the patient's AcroQoL scores were total score, 54.5\%; physical scale, 68.8\%; and psychological scale, $46.4 \%$, and subscale appearance and personal relationship score were $32.1 \%$ and $60.7 \%$ (Fig. 1). All of the patient's scores worsened compared with his previous AcroQoL scores despite having achieved normalized IGF1 levels. We suggested that he undergo SSRO with Le Fort I osteotomy to treat his mandibular 

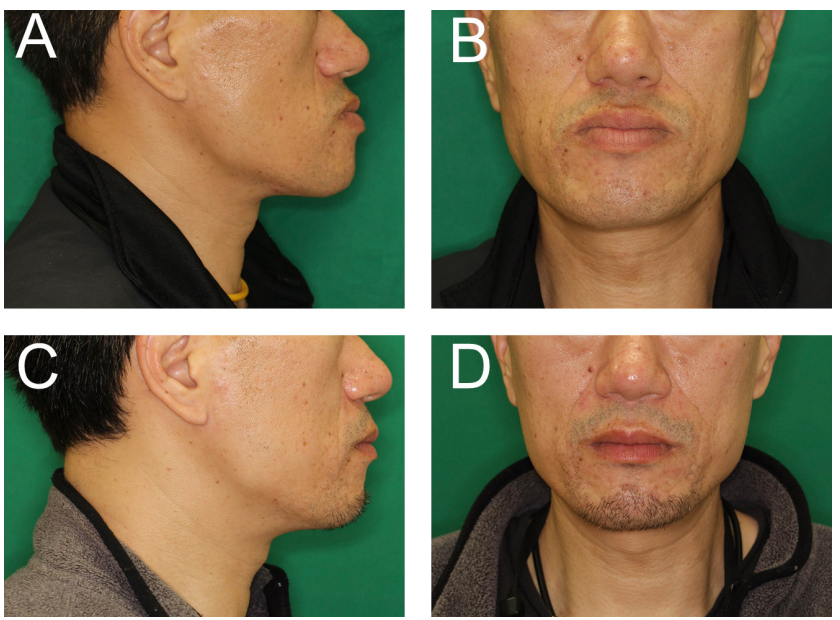

\section{Figure 2}

Facial photographs of the patient. Before (A, B) and after (C, D) sagittal split ramus osteotomy with Le Fort I osteotomy for mandibular prognathism.

prognathism and improve his AcroQoL scores. He was referred to the plastic and aesthetic surgeon, and lateral cephalometric radiographs and intraoral photographs showed massive mandibular prognathism (Fig. 3A and B). The patient's profilogram revealed SNA: $76.4^{\circ}$ (-1.8 s.D.), SNB: $84.9^{\circ}$ (+2.0 S.D.), ANB: $-8.5^{\circ}$ (-4.4 S.D.), mandibular
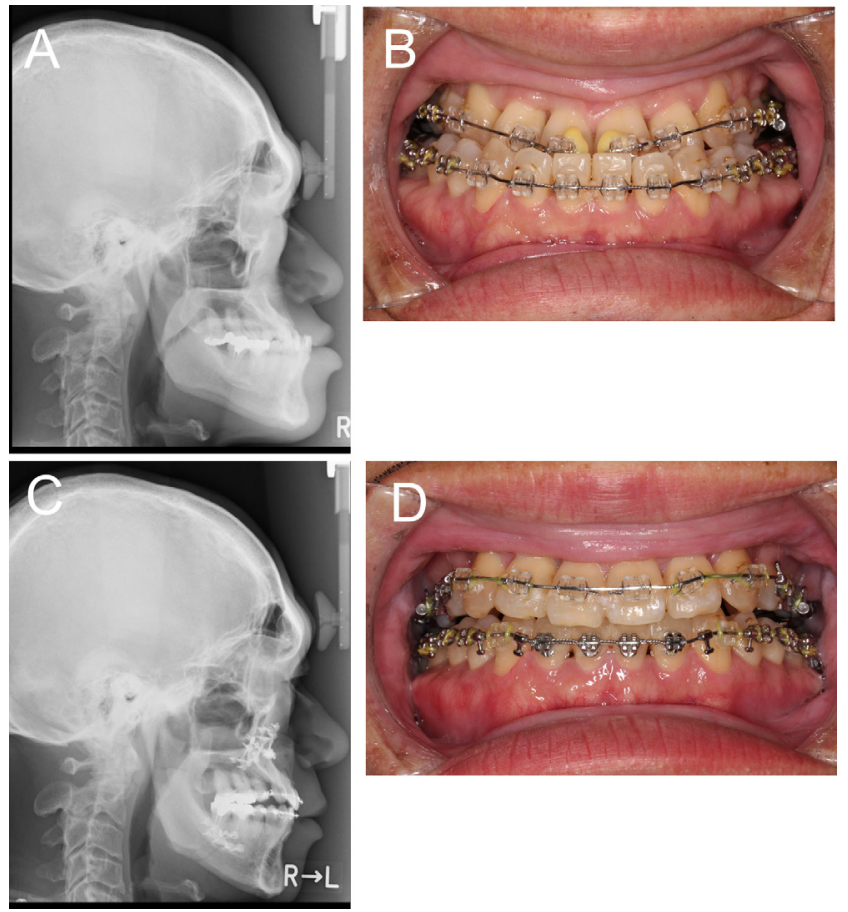

Figure 3

Lateral cephalometric radiographs and intraoral photographs of the patient before (A, B) and after (C, D) sagittal split ramus osteotomy with Le Fort I osteotomy for mandibular prognathism. plane angle: $21.5^{\circ}$ (-0.8 S.D.), gonial angle: $131.8^{\circ}(+3.5$ S.D.), angle of convexity: $-17.7^{\circ}$ ( -5.4 s.D.), facial angle: $98.4^{\circ}$ (+2.3 S.D.), angle grade 3 , U1-FH: $122.4^{\circ}$ (+2.4 s.D.), L1-MP: $86.8^{\circ}$ (-1.1 S.D.), and interincisal angle: $129.2^{\circ}$ (-0.1 s.D.) (Fig. 4). His facial bone was both thick and large (Fig. 5A and B). After 12 months of presurgical orthodontic treatment, the patient underwent SSRO with Le Fort I osteotomy (Figs 2C, 2D, 3C, 3D, 5C and 5D). During his operation, the maxillary first premolars were extracted, and his maxilla was divided into three sections in addition to Le Fort I. His maxilla was moved forward, and the maxillary dental arch was broadened.

\section{Outcome and follow-up}

After surgery, the patient's AcroQoL scores improved (total score, 62.5\%; physical scale, 65.6\%; psychological scale, 60.7\%; subscale appearance, $46.4 \%$; and subscale personal relationship, 75\%) (Fig. 1). Moreover, his HbA1c level decreased from $6.7 \%$ to $5.6 \%$ for 4 months after the osteotomy as tooth occlusion was corrected.

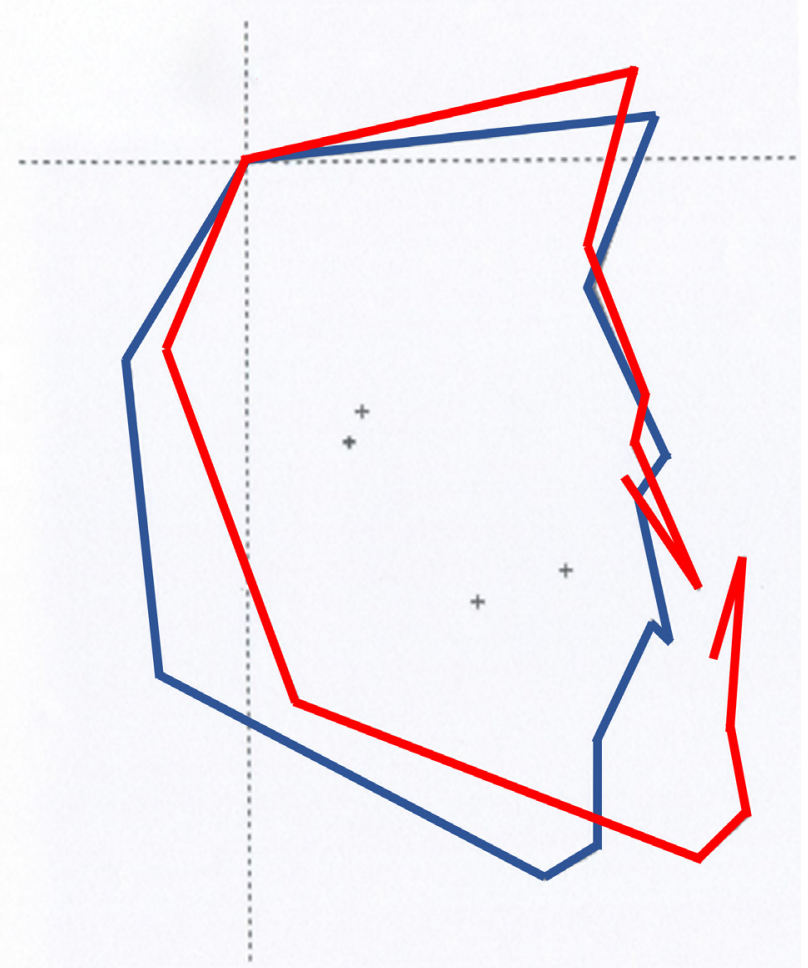

Figure 4

Profilograms of the patient in our case report (red line) and of a healthy subject (blue line). 

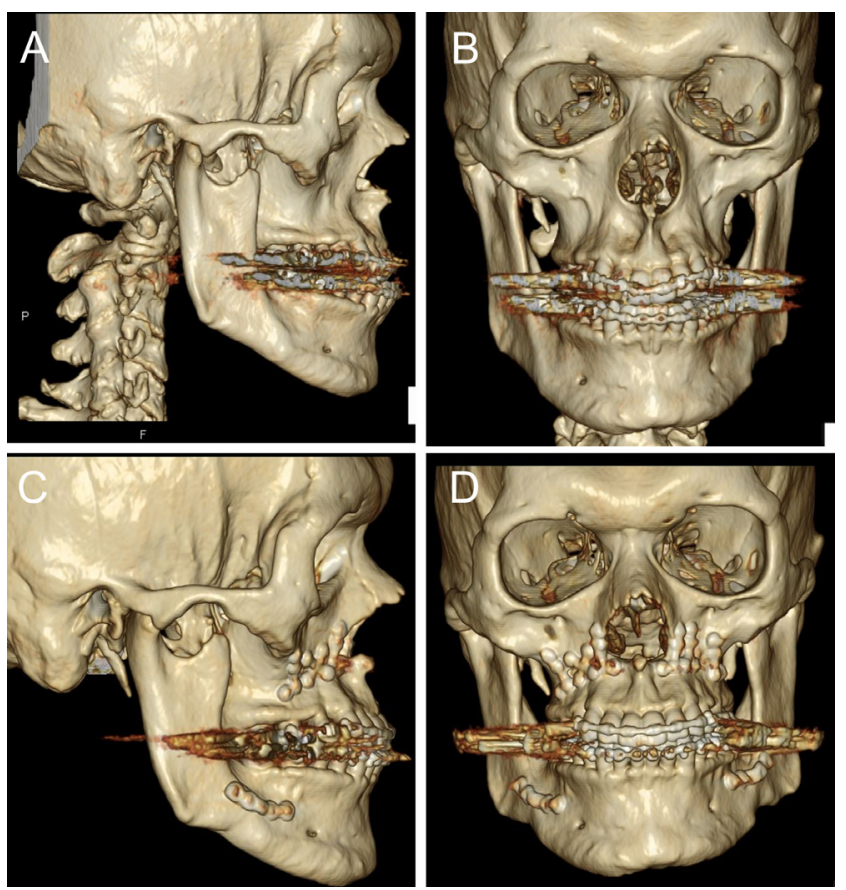

\section{Figure 5}

Facial CT scan of the patient before (A, B) and after (C, D) sagittal split ramus osteotomy with Le Fort I osteotomy for mandibular prognathism.

\section{Discussion}

In this case, we controlled our patient's serum IGF-1 level within the normal range with TSS and medication. Although his serum IGF1 level was normalized, his AcroQoL scores did not improve. Because the patient wanted to undergo surgery for mandibular prognathism, we decided to perform the operation. After the surgery, the patient's AcroQoL scores improved. Excess GH and IGF-1 secretions from the pituitary adenoma have both somatic and metabolic effects. The somatic effects include the stimulation of growth of many tissues, such as skin, connective tissue, cartilage, bone, viscera, and several epithelial tissues (1). The characteristic facial findings are macrognathia with enlargement of the nose and frontal bones and tooth spread. The most prevalent presenting complaints leading to diagnosis are acral change. After the treatment for pituitary adenoma and the excessive production of GH, patients with acromegaly must monitor coexisting conditions, such as changes in the face due to acromegaly. The problems regarding acromegalic face structure are related to not only cosmetic but also phonetic, chewing difficulties, and sleep apnoea. Previous reports have described successful treatments for an acromegalic face $(5,6,7)$.

After Le Fort I osteotomy is completed, the maxilla down-fracture technique is performed using digital pressure. However, in our patient, the maxilla was divided into three sections to widen the maxillary dental arch. An expected complication of this procedure is postoperative relapse of mandibular prognathism if the patient's GH and IGF1 levels increase. The mandibular angle of acromegaly is significantly greater than normal. The high incidence of oral manifestations relates to the duration of acromegaly (8), and oral complications are associated with the appearance of facial changes in patients with acromegaly.

Many patients experience a brief postsurgical depression as a result of adjusting to their new appearance (9). Patients who plan to undergo orthodontic surgery are classified by a psychological evaluation. Our patient was classified as high positive in the evaluation. We thought that he expected to be satisfied with the surgery.

Several scientific tools such as the 36-Item Short Form Survey, Sickness Impact Profile, and Nottingham Health Profile assess the QoL in patients (10). It is difficult to evaluate QoL because of the differences among diseases and their manifestations, but the AcroQoL is an acromegaly-specific QoL assessment tool. We evaluated our patient's AcroQoL scores before TSS and after normalization of serum IGF1 levels and found that his scores, especially the psychological score, worsened. Whether biochemical control improves the AcroQoL score is controversial $(11,12)$. Importantly, AcroQoL scores are lower in patients with persistent acromegalic activity compared to those with acromegaly in remission or in discordant remission (11). The appearance score is lower in Japanese patients who undergo pharmaceutical therapy than in those who are treated with surgery alone (13). Compared to no treatment, treatment with lanreotide showed worsening of all AcroQoL scores(14). The appearance score of our patient was also low even though he underwent TSS and injection therapies. The use of lanreotide, as in our patient, could result in lower psychological subscale appearance and personal relationship scores. Illness perception and treatment satisfaction are associated with QoL with acromegaly and the need for pharmaceutical therapy is associated with a depressive state (15). Our patient required drug treatment and may have been stressed about injection therapies. However, his AcroQoL scores after osteotomy for mandibular prognathism improved significantly, even though he continued injection therapies. In general, changes in facial appearance are only partially reversible even with normalization of growth hormone levels. In our case, osteotomy changed the facial appearance of our patient and he was satisfied with the result. His AcroQoL physical score only decreased after the osteotomy. We 
suppose that his muscle strength weakened as his GH and IGF1 levels normalized and lean body mass decreased. It is difficult to estimate the degree of dissatisfaction that patients with acromegaly experience with respect to their appearance. However, the AcroQoL helps to understand the challenges faced by these patients and provide appropriate support for an improved QoL.

In conclusion, this case report shows a distinct benefit of osteotomy for a patient with acromegaly and confirms patient satisfaction using the disease-specific AcroQoL.

\section{Declaration of interest}

All authors declare that there is no conflict of interest that could be perceived as prejudicing the impartiality of the research reported.

\section{Funding}

This work was supported by MHLW FC Program Grant Number 20 FC0201.

\section{Patient consent}

Written informed consent has been obtained from the patient for publication of the case report and the accompanying images.

\section{Author contribution statement}

$\mathrm{TT}$ and KT were responsible for case description, literature review, writing and editing; SK is an oral and maxillofacial surgeon. The authors would like to thank Enago (www.enago.jp) for the English language review.

\section{References}

1 Melmed S. Medical progress: acromegaly. New England Journal of Medicine 2006355 2558-2573. (https://doi.org/10.1056/ NEJMra062453)

2 Biermasz NR, van Thiel SW, Pereira AM, Hoftijzer HC, van Hemert AM, Smit JW, Romijn JA \& Roelfsema F. Decreased quality of life in patients with acromegaly despite long-term cure of growth hormone excess. Journal of Clinical Endocrinology and Metabolism 2004 89 5369-5376. (https://doi.org/10.1210/jc.2004-0669)

3 Webb SM, Prieto L, Badia X, Albareda M, Catalá M, Gaztambide S, Lucas T, Páramo C, Picó A, Lucas A, et al. Acromegaly Quality of Life Questionnaire (ACROQOL) a new health-related quality of life questionnaire for patients with acromegaly: development and psychometric properties. Clinical Endocrinology 200257 251-258. (https://doi.org/10.1046/j.1365-2265.2002.01597.x)

4 Katznelson L, Laws ER, Jr, Melmed S, Molitch ME, Murad MH, Utz A, Wass JA \& Endocrine Society. Acromegaly: an endocrine society clinical practice guideline. Journal of Clinical Endocrinology and
Metabolism 201499 3933-3951. (https://doi.org/10.1210/jc.20142700)

5 Tornes K \& Gilhuus-Moe O. Correction of jaw deformities subsequent to treatment of acromegaly. International Journal of Oral and Maxillofacial Surgery 198615 446-450. (https://doi.org/10.1016/ s0300-9785(86)80036-9)

6 Yagi T, Kawakami M \& Takada K. Surgical orthodontic correction of acromegaly with mandibular prognathism. Angle Orthodontist 2004 74 125-131. (https://doi.org/10.1043/0003-3219(2004)074<012 5:SOCOAW >2.0.CO;2)

7 Smith CB \& Waite PD. Surgical management of obstructive sleep apnea in acromegaly with mandibular prognathism and macroglossia: a treatment dilemma. Journal of Oral and Maxillofacial Surgery 201270 207-210. (https://doi.org/10.1016/j. joms.2011.05.022)

8 Herrmann BL, Mortsch F, Berg C, Weischer T, Mohr C \& Mann K. Acromegaly: a cross-sectional analysis of the oral and maxillofacial pathologies. Experimental and Clinical Endocrinology and Diabetes : German Society of Endocrinology 2011119 9-14. (https://doi. org/10.1055/s-0030-1255020)

9 Frost V \& Peterson G. Psychological aspects of orthognathic surgery: how people respond to facial change. Oral Surgery, Oral Medicine, and Oral Pathology 199171 538-542. (https://doi.org/10.1016/00304220(91)90357-i)

10 Busija L, Pausenberger E, Haines TP, Haymes S, Buchbinder R \& Osborne RH. Adult measures of general health and health-related quality of life: medical Outcomes Study Short Form 36-Item (SF-36) and Short Form 12-Item (SF-12) Health Surveys, Nottingham Health Profile (NHP), Sickness Impact Profile (SIP), Medical Outcomes Study Short Form 6D (SF-6D), Health Utilities Index Mark 3 (HUI3), Quality of Well-Being Scale (QWB), and Assessment of Quality of Life (AQoL). Arthritis Care and Research 201163 (Supplement 11) S383-S412. (https://doi.org/10.1002/acr.20541)

11 Trepp R, Everts R, Stettler C, Fischli S, Allemann S, Webb SM \& Christ ER. Assessment of quality of life in patients with uncontrolled vs. controlled acromegaly using the Acromegaly Quality of Life Questionnaire (AcroQoL). Clinical Endocrinology 200563 103-110. (https://doi.org/10.1111/j.1365-2265.2005.02307.x)

12 Roumeau S, Thevenon J, Ouchchane L, Maqdasy S, BatisseLignier M, Duale C, Pham Dang N, Caron P, Tauveron I \& Devoize L. Assessment of oro-dental manifestations in a series of acromegalic patients, the AcroDent study. Endocrine Connections 20209 824-833. (https://doi.org/10.1530/EC-20-0176)

13 Yoshida K, Fukuoka H, Matsumoto R, Bando H, Suda K, Nishizawa H, Iguchi G, Ogawa W, Webb SM \& Takahashi Y. The quality of life in acromegalic patients with biochemical remission by surgery alone is superior to that in those with pharmaceutical therapy without radiotherapy, using the newly developed Japanese version of the AcroQoL. Pituitary 201518 876-883. (https://doi.org/10.1007/ s11102-015-0665-2)

14 Hua SC, Yan YH \& Chang TC. Associations of remission status and lanreotide treatment with quality of life in patients with treated acromegaly. European Journal of Endocrinology 2006155 831-837. (https://doi.org/10.1530/eje.1.02292)

15 Sievers C, Dimopoulou C, Pfister H, Lieb R, Steffin B, Roemmler J, Schopohl J, Mueller M, Schneider HJ, Ising M, et al. Prevalence of mental disorders in acromegaly: a cross-sectional study in 81 acromegalic patients. Clinical Endocrinology 200971 691-701. (https://doi.org/10.1111/j.1365-2265.2009.03555.x)
Received in final form 6 March 2021

Accepted 12 April 2021 